\title{
Mathematical Tools and Methods for Analysis of SDN: A Comprehensive Survey
}

\author{
Girish L \\ Research scholar, \\ CIT, Gubbi \\ Karnataka, India \\ girish.1@cittumkur.org
}

\author{
Dr. Sridhar K N Rao \\ Professor, \\ CIT, Gubbi \\ Karnataka, India \\ sridhar@cittumkur.org
}

\begin{abstract}
Software defined Networking (SDN) is one of the developing networking technologies which is attracting extreme interest from both industry and research groups. SDN's application is mainly found in datacenters and research network environments. SDN is a technology which controls the whole network using software that adapts the behavior of the network to be compatible with various applications and environments. In this paper, our goal is to help researchers by providing an insight about the various mathematical tools and verification methods which can be used in the analysis of Software defined networking. These mathematical tools and verification methods help in formalizing the OpenFlow protocol. We assure researchers that by making use of these mathematical tools and verification methods the formal guarantees in the analysis of SDN will not be violated. We present performance prediction tools like Queuing theory and Network Calculus that assists network planners to study how the networks' performance gets affected by varying traffic loads and as the network utilization changes.
\end{abstract}

Keywords-Queuing theory, Network Calculus, SDN, Process Algebra

\section{INTRODUCTION}

The Computer network is a collection of interrelated hardware components like routers, switches, firewalls etc. Entire network services are dependent on these registered hardware appliances that are distributed and various purposes. With the aspiration to gain a high quality data plane, this custom has typically sustained, with such precise and specific hardware systems being mounted also for typical software functions like substitutions, firewalls, and caches.

The real problem with this is its dearth of supporting addition of services and network apprising process. To overcome these disputes and to diminish the capital expenditure, virtualization has arisen as a method to decouple the software networking processing and applications from their supported hardware and permit network services to be implemented as software [1], [2].

By applying virtualization techniques that allow appropriate management of dynamically produced server instances, the cloud data centers have amplified their efficiency and flexibility.

The application of network function virtualization (NFV) over software defined Networking (SDN) has given rise to an analogous revolution in both WAN (Wide area networks) and in the network of data centers.
NFV does the transformation of networks from varied purpose hardware appliances into software executing in VMs and containers [5] on common ready-made hardware to lessen the cost expenses [6], [7]. With this transformation to software based atmospheres by NFV network services are not difficult to deploy, and extra powerful and supple, supporting more composite topologies and networks comprising of rich featured resident functions compared to hardware based implementations. It is accessed by wired as well as wireless network platforms. NFV decreases principal outflows of both capital expenditure and operational expenditure by lessening the procurement of the exclusive hardware machines, their power and cooling necessities [6].

Software defined networking (SDN) [3], [4] is a prototype of networking, gives confidence to overcome disadvantages of existing network organizations. It uses a centralized supervisor to take care of stream of packets in the data plane through southbound interface OpenFlow protocol [12], 13]. This new methodology makes network management easier, has capability to save expenses for the organization. Based on OpenFlow a numerous SDN applications from hardware to software retailers are installed in dissimilar kinds of networks, from trivial organizations to huge data centers. This increases the number of trials done with SDN-enabled networks. As a result, it produces additional challenges, because queries regarding SDN performance and scalability are not yet examined. There are very less number of performance evaluation studies [14], [15] on SDN and OpenFlow architectures. Even though simulation trainings and investigations are amongst the most extensively used performance assessment techniques, analytical modeling has got particular profits as well.

Numerous mathematical tools are present for the analyses, formalization, modeling and verification of both SDN. The paper presents a detail survey of such approaches and their shortcomings and later, it gives an assessment of mathematical tools for various situations/scenarios of SDN.

The structure of the report is as follows. The Section II covers a thorough survey of mathematical methods which are used in SDN. Section III presents a list of mathematical tools that are used for verification in the era of SDN. 


\section{RELATED WORK}

This section presents a brief survey of various mathematical approaches that can be applied on various disputes of software defined networking. We begin with the discussion of different analytical performance model of computer network. Then, we present the summary on mathematical tools/methods for specifications and verification of network protocols. Fig.1 illustrates the summary of the review work categorized according our taxonomy.

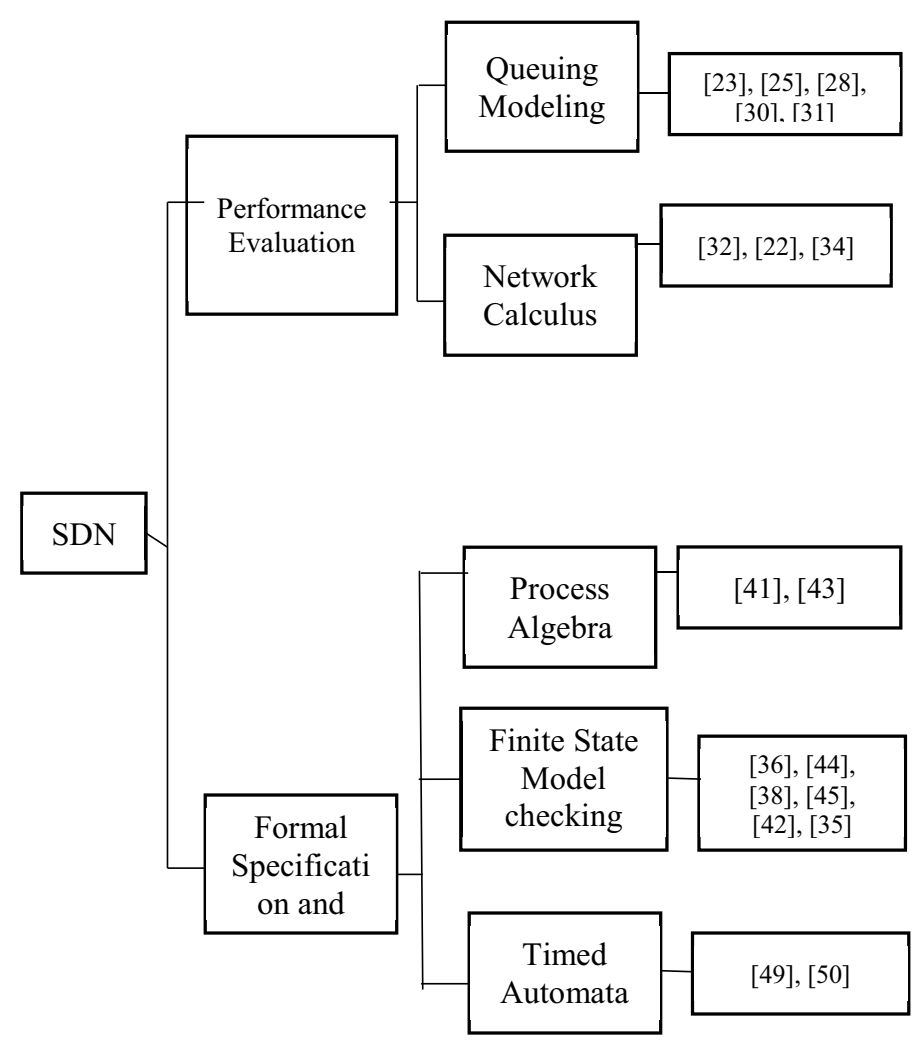

Fig. 1 Review work categorization according our taxonomy.

\section{A. Performance Evaluation using Analytical Modeling}

Two key methods are used for the development of analytical performance model for computer networks: Queuing theory [16] and network calculus [17].

\section{1) Queing theory}

Queuing theory is a well-established division of analysis regularly used and more suitable for networks related studies [22]. Using these mathematical models on the current networks helps in providing an intuition about the performance variations with the change in traffic volumes. Queuing theory studies capabilities in a diversity of network traffic situations and proposes a collection of tools permitting the description of network performance.
Queuing network modelling [23], is a specific methodology to computer system modelling in which the computer system is characterized as a network of queues which is assessed analytically. A network of queues is a group of service centers, which signify system resources, and clients, which represent operators or transactions. Analytic evaluation includes using software to solve competently a set of equations persuaded by the network of queues and its parameters.

Queuing theory modelling methods contributes to a precise performance assessment of the production networks grounded on SDN that are visible to fresh customer demands repeatedly. A very few performance evaluation studies are found on software defined networking and network function virtualization architecture. We analyse a number of research works concentrating on addressing performance and scalability concerns at switch [18] [21] and controller [19] [20].

\section{a) Queuing model for OpenFlow Switches:}

Queuing theory used in OpenFlow switches for the process of packet arrival, packet forwarding and characterizing the packet forwarding process with the Queuing model $M X=M=1$. Till now, most of the analytical techniques based on OpenFlow networks postulate that packet traffic in a switch leads to the Poisson distribution [28]. Though the studies carried out so far pointed to the fact that packets arriving in networks emulates running trains [24] [25] instead of Poisson stream [26].

Bing Xiong [27], describes about the packet forwarding technique in OpenFlow switch. When the packet is delivered it is the switch that places packet into the packet queue at the entrance port. The switch takes the packet from its packet queue and accesses the corresponding information with every protocol header to calculate the flow key. In turn, key is utilized to match the entries against the stream tables. In case the match fails, the switch suspends the packet and hands over the entire packet to its corresponding SDN controller. With the supposition of adequate packet buffer to all entrance queues of the switch, the performance of the packet forwarding technique of the OpenFlow switch can be described by way of a $M X=M=1$ queue.

\section{b) Queuing model for SDN Controller}

OpenFlow switch executes flow table lookups on the arrival of a packet. If the lookups do well, the switch applies the activities in the matched table entry to the packet, classically accelerating it to the identified interface. Else, the packet has to fit in to a different flow, and the switch directs it in an arriving packet message to a higher SDN controller. Then the controller governs the consistent stream instruction and directs the packet flowing out or flow mod message for switch. As significance, a SDN controller accepts a stream of packet arriving messages from every OpenFlow switch.

OpenFlow architecture can be modelled as forward Queuing technique by $\mathrm{M} / \mathrm{GI} / 1$ and a feedback Queuing technique with the delay loss type M/GI/1- S. It intentionally starts by presumptuous Markovian servers of together for 2 systems, i.e. an $\mathrm{M} / \mathrm{M} / 1$ used aiming at forward technique and $\mathrm{M} / \mathrm{M} / 1$ - $\mathrm{S}$ used aiming at feedback technique, to assess the toughness of the modeling methodology. 
For the forward system, arrival procedure on the switch is grouping in entering procedure of packets obtained through line cards having rate of $\lambda$ and packets that advanced from switch buffer once the controller has found applicable act and conforming access was produced found in flow table. Hence the OpenFlow controller can be demonstrated through feedback using $\mathrm{M} / \mathrm{M} / 1-\mathrm{S}$ queuing system.

Numerous studies have designated that packet arrival procedure is not a Poisson stream because of the unexpectedness and perseverance of network traffic, but stream emergence process follows to Poisson distribution [28]. This means that the Poisson method is appropriate for the sequence of the first packets in all streams. In SDN architecture, an OpenFlow switch only directs the first packet of a flow in an arriving packet message from switch to its controller. Accordingly, the arriving packet messages from a switch to its controller emulate the Poisson distribution, and messages of the arriving packet at a SDN controller is thought to be a hybrid of multiple Poisson streams.

Once packet message arrives, controller regulates the corresponding flow rule by observing up its inner forwarding tables regularly known as forwarding information base (FIB). If the lookup flops, the controller alternatives to its topology detection and path calculation to learn and process the message. Henceforth, the dispensation time of arriving packet messages in controller can be thought to adapt to negative exponential distribution having the parameter $\mu$, which signifies the probability of the message processing rate.

Xia Peng [29], demonstrated the concise Queuing Model $M / M / 1$ to describe the arriving packet message dispensation of a SDN controller. Through the above Queuing model, the length of packet message queue at a SDN controller $N(t)$ is a birth and decease process on the countable infinite state set $\mathrm{E}=$ $\{0,1,2, \ldots\}$.

Kashif Mahmood [30], proposed an analytical model for an OpenFlow empowered SDN based on Jackson network. The model is precise even for the case when the possibility of new flows is quite large. The applicability of the model is understood by establishing two performance procedures, the average packet sojourn time and the distribution of time consumed by a packet in the network, by means of the proposed model. Secondly, we presented by a toy example that the model can be readily stretched to more than one switch in the data plane.

\section{c) Queing model for Distributed SDN controller}

SDN control plane can be made scalable if it can handle additional network event requirements with growing complexity and at the same time maintaining good quality of service. To expand the scalability property of SDN control plane, Soheil Hassas Yeganeh [8], proposed two methodologies. The first is to transfer certain control operations belonging to controller to switches, this helps to decrease event requirements submitted to the controller considerably. The second methodology is to distribute load among several controllers by implementing distributed architecture for the control plane. There are numerous distributed architectures for control plane of SDN, to list a few, Onix [9], Hyperflow [10], Kandoo [11].

There are various strategies which can be adopted to understand the multiple controllers' methodology: decentralized and hierarchical structures. In case of decentralized methodology, controllers are flat structured. Two approaches are found in decentralized method. Each controller takes only the topology of its local networks. For hierarchical method, switches and controllers are arranged in the form of a tree. Controllers are allocated in two dissimilar layers. Each switch is a leaf node. Leaf controllers and internal controllers are the two controllers in the tree. A leaf controller is responsible for all the switches in the sub tree. It is not containing by any other controller in the sub tree.

Logically integrated root controller and Multiple native controllers control the entire network. These controllers together form distributed control plane. Every switch will be monitored through a single local controller. Multiple numbers of switches are monitored by every local controller. Whenever root controller wants to set up the flow patterns on switches, it hands overs the requests to the corresponding local controller.

Guodong Wang [29], presented a Queuing model for multiple controllers' approach. Local controllers modeled as a $\mathrm{M} / \mathrm{M} / 1 / \mathrm{m}$ system which is implemented in [30]. The root controller is regarded as an $\mathrm{M} / \mathrm{M} / 1$ system. The root controller is generally running in a more dominant server with a larger memory, so it is sensible to assume the buffer size is infinite. In addition, decided as the infinite buffer size can make simpler the simulation when cascading the $M / M / 1$ and $M / M / 1 / m$ system by using the Kleinrock model [31].

\section{2) Network Calculus}

This segment comprises a brief overview of network calculus, meanings, system models, exploration of SDN switch, communication between switches and SDN controllers. The scientific explanation of size of the queue, interval restricted SDN switches, buffer necessities of SDN switches and controllers in a deployment are explained in this segment.

Network calculus is used to control the flow complications in networks. Primarily the worst-case perspective. As a framework, it is used to develop deterministic agreements on deferment, outputs, queue lengths, and other analogous performance metrics.

Mathematically Network calculus is grounded on min-plus algebra. It is understood in the form of system deterministic theory and stochastic Queuing systems as well. Network Calculus is facilitated in both deterministic [32] and stochastic [33] variations.

Stochastic network calculus is used to examine performance assurance in information systems [21], [22]. It has its basics in the min-plus as well as max-plus convolution Queuing principles, and it has marvelous prospective concerned with Queuing type problems.

It matches classical Queuing theory [21]. In [22], Ciucu et al. discuss sharp bounds in stochastic network calculus. Stochastic network calculus can be used to compute each flow Queuing system metrics in a cohesive manner for a huge group 
of scheduling algorithms. Additionally, each flow outcomes are stretched in direct manner. Starting at single queue up to huge class of Queuing networks that are agreeable.

Siamak Azodolmolky [34] in his work on SDN says that, conferring to the parameters set for the cumulative arrival process, the SDN switch model seized the locked system of the packet deferment and buffer size in the SDN switch. Additionally, an analytical model was used to analyze the interface among SDN switches and SDN controller. If constraints of increasing incoming processes and operability of the flow control for SDN controller are prearranged, the network designer will be capable of calculating the higher bound approximation of packet interval and buffer constraints in SDN switches. Network calculus for SDN utilizes traffic requirements to model the incoming and topmost features of a flow. Below given is brief description of Network calculus for SDN switch and Controllers.

\section{a) Network calculus for SDN switch}

Switch is a network component. It consists of input $A$, a flow table control for input $C$, and an output $F$ where,

$F=C(A(t))$,

$A(t)$ : the increasing number of entering network by time $t$,

$C(n)$ : total number of packets arriving, whose movement is controlled (example: "Flow Mod" process) between first $n$ packets arriving

$F(t)$ : the increasing number of packets departures in time $t$.

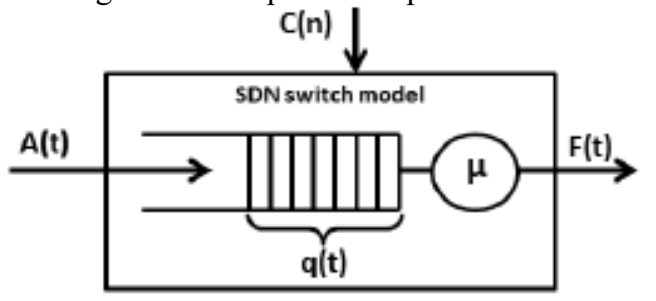

Put it in the other way, the collective count of packets coming out by time $t$ is same as increasing count of packets coming to switch, and their movement is monitored by the SDN controller for the duration of time $t$. The consecutive packets, corresponding to the flow table entry, are not progressed to the OpenFlow controller if procedure in the flow table of the switch is stored by the OpenFlow controller.

\section{b) Network Calculus for SDN controller}

The principle of network calculus on analytical model proposes a locked form of upper bounds for queue lengths in the SDN switch and SDN controller. The figure below shows the interface between an SDN controller and SDN switches. According to multiplexing rule, both the input packets of other SDN switches along with the SDN switch illustrated in figure above can be taken into account. Increasing incoming process A2 signifies the packet coming through additional SDN qualified switches; in turn these are managed by the centralized controller.

\section{B. Formal Specification and Verification}

The communication protocol implementations suffer from extremely unpredictable and complicated behaviour. The correctness of this protocol can be achieved neither by conducting testing nor by informal reasoning. Hence it is becoming crucial to go with formal verification techniques. These formal models can be successfully adopted in specifying and verifying the propertied of the communication protocols. In the following section we describe some of the Specification and verification techniques for communication protocols.

In case of a protocol having formal specifications, the primary step to be guaranteed is that the specification is free from errors. The identification of errors can be carried out in two processes: Verification and Validation. Validation is the process identifying the syntactic errors.

Verification is a process of identifying the semantic errors with respect to the correctness of the protocol. It is just a process to assure that the object is as per the specifications and the behavior is as expected.

\section{1) Formal Specification for SDN}

This section presents some specification techniques for software defined network protocols. Formal methods are mathematics-based techniques to specify, develop, and verify software and hardware systems, and are expected to increase reliability and robustness of the system.

A formal specification is nothing but the language comprising of formally defined semantics, vocabulary as well as syntax. These specification languages must be grounded on logic, mathematics but not natural languages.

SDN network operators and application/service providers informally develop an abstract network model of desired properties. After that they design the formal specification for the properties that verifies the implementation and fulfills these properties.

Overall, the old methods used for understanding the network protocols and devices are grounded on public agreements of informal specification. These old methods can be improvised by adopting the formal methods used for the development process for SDN. The objectives of the formal specifications include conceptual model of components, logical switch/router models, tools and mechanisms for SDN, userdefined topologies of virtual networks, network protocols etc. It is also possible to encode the informal specifications of these objectives to formal specification to reflect the features of these objectives among the present methods for formal specification.

After describing the specifications formally, the protocol engineers can verify for the presence of any inconsistencies and faults in the specification with the assistance of experts or tools. Any formal verification method can be employed to carry out validation and verification process. But each method has its own positives and negatives.

Here we try to describe the formal specifications with respect to virtual network topology of SDN. In this regard, the two closely related research works carried out are NetCore and NDlog each with different viewpoints. NetCore (Network Core Programming Language) is used to express the packetforwarding policies and consists of formal semantics. NDlog (Network Datalog) is a distributed recursive query language useful for executing the network graphs queries. 


\section{2) Formal Verification for $S D N$}

The main problems arising in the operation of SDN are associated with lack of standardized communication protocols, the presence of inaccuracies and inconsistencies in the existing version of the specification. Lack of uniform standards significantly affects the SDN design. Developers need to consider their own formalization and implementation, its decisions all the requirements laid down in the various versions of the OpenFlow protocol.

Even though it is a challenging task, the network administrators are themselves responsible for fixing the bugs discovered in the OpenFlow programs developed by them. Hence there should be some methods to check the precision of OpenFlow networks automatically. Usually the traditional tools like ping and traceroute are used to check the correct working of networks. Because the networks are intrinsically asynchronous and distributed it becomes challenging to guarantee that the network is working fine. Even the OpenFlow networks are suffering from the same problem. Hence to check the correctness of the networks even more tough and efficient methods are in need.

Thus, before the integration of the protocol in the complex distributed system is necessary to control (check) protocol implementation conformance to all requirements described in the specification [39].

$\mathrm{SDN}$ is now taking a step in trying the formal methods to check the correct working of networks. Definitely lots of efforts been spent to evaluate the correctness of SDN programs using finite state models.

\section{a) Fininte-state model checking of SDN programs}

Tools such as NICE [36] uses finite-state model to check the precision of SDN controllers. The SDN program in the form of a state-transition system emulates with respect to events. A tangible network topology is also unambiguously demonstrated. There are many advantages of Finite state model checking. There is no need of inductive invariants and employs easiest verification methodology. However, it can never assure for the absence of bugs in the SDN programs. But still without any difficulty it can be used for arbitrary programs. Finite state model checking is also not scalable with realistic networks. NICE system takes long time for completing the model checking.

Finite-state model checking is also used for examining the precision of SDN programs over huge networks [37]. Another system called FlowLog [38] applies Finite-state model by restricting number of packets delivered. Also it is observed that for few network arrangements these restrictions will not produce accurate results.

SDPOR-DS [45], is another system whose objective is to evaluate the distributed systems like OpenFlow networks. It is mainly used to diminish the difficulty of state-explosion. This is achieved by immense arrangement of state transitions and cropping examination of state space.

By comparing the performance of SDPOR-DS with NICE, a state-of-the-art tool used for model checking of OpenFlow systems has been presented. With NICE system it has consumed only $31 \%$ of execution time.
Aaron Gember [35], for the first time presented a system called VeriCon to check whether the SDN program is working fine for all possible network arrangements and on all probable structures of network events. VeriCon system assures only either the controller program works fine on all possible network arrangements or gives a solid counterexample. It follows the first order logic to identify all acceptable network arrangements and desired network wide invariants. It rapidly verifies the correctness, discover the bugs of moderate core SDN programs.

FLOVER [42], is a model testing system. FLOVER [42], a model testing system. It confirms whether the cumulative assessment of flow strategies inside OpenFlow network violates network's security policy. It is developed using Yices SMT solver [43], in turn it is assimilated with OpenFlow network controller. FLOVER authenticates a group of identified nonbypass specifications in contrast to the modernized flow instruction set every time it distributes recently produced flow guidelines to an OF-switch.

FLOVER either updates or adds command containing minimum of one flow rule, and then evaluates already defined non-bypass properties in contrast to the set of produced rules and formerly dedicated flow rules if it gets a flow rule appeal through an OF-switch.

Amer Tahseen [40], proposed Algebra of communicating resources(ASCR) [41] as mathematical tool for verification of OpenFlow protocol. ASCR was established for formal checking of real-time embedded systems. ACSR is attempt to verification of distributed complex structures that are delicate to deferment, deadlines, and process interface and resource accessibility

Cbench [44] is benchmark tool programmed in PERL and $\mathrm{C}$ languages and used for analysis of OpenFlow controllers by producing events of the arriving packets for new traffic. Cbench exactly matches the cluster of switches that associate with a controller, send the packet messages and lookout for flow-mods to acquire pushed down.

Oflops [48] stands for OpenFlow Operations per Second. It is a separate controller tool written in $\mathrm{C}$ language that benchmarks several features of an OpenFlow switch. Oflops develops an integrated framework for assembling and executing operation uncertain examinations to enumerate a switch's enactment. It is an open source framework for testing OpenFlow controllers.

OFRewind is a tool used for testing and debugging in the network at multiple levels. It is mainly used to reproduce the software bugs [46]. With this tool, it is possible to record traffic (both control and data) in the network, and rerun it at an adaptive rate with adequate accuracy at multi-granularity. OFRewind performs as a clear substitution between the controller and the switches in the OpenFlow network. Through policy specification language, it is possible to tune OFRewind to record precise subpopulations of traffic and replay over a different OpenFlow network. 


\section{b) Fininte Timed Automata}

Timed automata models are the extension automata models. The real time limitations are embedded to node and edge labels. Through handshake models, synchronizations are carried out in an asynchronous manner. The important characteristic of timed automata is its capability of checking and updating the real valued timers. For example, using time restrictions on node invariants, using them as the transition guards, using clocks in global context by increasing all of them with the same value at the same time and set the timers to zero.

The tool UPPAAL [49], uses formal specification and validation of SDN in the form real time system. By making use of UML diagram editor Dia it gives a UML grounded depiction for SDN. The method used for SDN verification, is grounded on the transformation of SDN depiction into network for timed automata. The tool UPPAAL makes use of networks related to finite timed automata.

\section{TOOLS}

In this section, a comparison review of the verification tools is presented, these can be found on Fig. 2 .

\begin{tabular}{|c|c|c|c|}
\hline $\begin{array}{c}\text { Features/ } \\
\text { Tools }\end{array}$ & Purpose & GUI & Description \\
\hline UPPAL & $\begin{array}{l}\text { Modeling, } \\
\text { simulating and } \\
\text { verifying real } \\
\text { time system }\end{array}$ & Yes & $\begin{array}{l}\text { Allows user to analyze } \\
\text { networks for timed } \\
\text { automata }\end{array}$ \\
\hline NICE & $\begin{array}{l}\text { To check the } \\
\text { correctness of } \\
\text { controller }\end{array}$ & Yes & $\begin{array}{l}\text { Its main goal is to test } \\
\text { computer program } \\
\text { without requiring any } \\
\text { type of modification }\end{array}$ \\
\hline FLOVER & $\begin{array}{l}\text { Model checking } \\
\text { for security } \\
\text { aspects }\end{array}$ & Yes & $\begin{array}{l}\text { Provides correct and } \\
\text { automatic method for } \\
\text { verifying security } \\
\text { properties of OF } \\
\text { controller } \\
\end{array}$ \\
\hline CBENCH & $\begin{array}{c}\text { Evaluate } \\
\text { openflow } \\
\text { controllers }\end{array}$ & Yes & $\begin{array}{c}\text { Used to emulate } \\
\text { openflow switches } \\
\text { which are generate } \\
\text { workload to controller }\end{array}$ \\
\hline $\begin{array}{c}\text { VERICO } \\
\mathrm{N}\end{array}$ & Verify SDN apps & Yes & $\begin{array}{l}\text { Used for correctness od } \\
\text { SDB apps in large } \\
\text { scale. }\end{array}$ \\
\hline $\begin{array}{l}\text { FLOW- } \\
\text { GUARD }\end{array}$ & $\begin{array}{c}\text { Verify SDN } \\
\text { policy }\end{array}$ & No & $\begin{array}{c}\text { Detecting and resolving } \\
\text { firewall policy } \\
\text { violation of openflow } \\
\text { switches }\end{array}$ \\
\hline
\end{tabular}

Fig. 2 Comparison review of verification Tools

\section{CONCLUSIONS}

Since SDN is a booming research area and has a lot of scope to dig, it is becoming crucial for the researchers to analyse the performance of SDN by making use of well sophisticated mathematical tools. In this view we presented the two cornerstones of network performance analysis, Queuing Model and Network calculus. Though Queuing Model and Network calculus are the well-established branches for network analysis used frequently, according to the survey it is observed that very little work has been done in the area of SDN analysis using Queuing Model and Network calculus. In this paper we made an effort in presenting a brief review on how Queuing model can be used for evaluating OpenFlow Switches, SDN Controllers and Queuing Distributed SDN Controller and how Network calculus can be used for SDN switches and for SDN controllers. We also presented a Formal Specification and Verification for SDN.

We discussed about the importance of NICE tool and how it uses the finite-state model checking to confirm the precision of SDN controllers. We hope this effort helps the researchers in understanding the importance of mathematical tools in analyzing SDN and helps them to come out with new innovative thoughts and ideas in the field of SDN.

\section{REFERENCES}

[1] G. Schaffrath et al., "Network virtualization architecture: Proposal and initial prototype," in Proc. 1st ACM Workshop Virtualized Infra struct. Syst. Archit., 2009, pp. 63_72.

[2] N. M. M. K. Chowdhury and R. Boutaba, "A survey of network virtualization," Computer Network, vol. 54, no. 5, pp. 862_876, 2010.

[3] Diego Kreutz, Fernando M. V. Ramos, Paulo Esteves Ver1'ssimo, Christian Esteve Rothenberg, Siamak Azodolmolky, Steve Uhlig "Software-Defined Networking: A Comprehensive Survey", Vol. 103, No. 1, January, IEEE, 2015, pp 4-75

[4] B Raghavan et al., "Software-defined internet architecture: Decoupling architecture from infrastructure,' in Proc. 11th ACM Workshop Hot Topics Netw., 2012, pp. 43-48.

[5] Yukihiro Nakagawa, Chunghan Lee, Kazuki Hyoudou, Shinji Kobayashi, Osamu Shiraki, Jun Tanaka, "Dynamic Virtual Network Configuration between Containers using Physical Switch Functions for NFV Infrastructure", IEEE Conference on Network Function Virtualization and Software Defined Network (NFV-SDN), 2015.

[6] M. Casado et al., "Virtualizing the Network Forwarding Plane," Proc. Wksp. Programmable Routers for Extensible Services of Tomorrow, New York, NY, 2010, pp. 8:1-6.

[7] J. Martins et al., "ClickOS and the Art of Network Function Virtualization," 11th USENIX Symp. Networked Systems Design and Implementation, Seattle, WA, 2014, pp. 459-73.

[8] Soheil Hassas Yeganeh, Amin Tootoonchian, and Yashar Ganjali, University of Toronto, "On Scalability of SoftwareDefined Networking”, IEEE 2013

[9] Teemu Koponen, Martin Casado, Natasha Gude, Jeremy Stribling, Leon Poutievski, "Onix: A Distributed Control Platform for Large-scale Production Networks", ACM 2010.

[10] A. Tootoonchian and Y. Ganjali, "Hyperflow: a distributed control plane for OpenFlow," in Proceedings of the 2010 internet network management conference on Research on enterprise networking. USENIX Association, 2010, pp. 3-3.

[11] S. Hassas Yeganeh and Y. Ganjali, "Kandoo: a framework for efficient and scalable offloading of control applications," in Proceedings of the first workshop on Hot topics in software defined networks. ACM, 2012, pp. 19-24.

[12] Casado M, Freedman M J, Pettit J, Luo J, McKeown N, Shenker S. "Ethane: taking control of the enterprise". ACM SIGCOMM Computer Communication Review, 2007, 37(4): 1-12.

[13] Benamrane F, Mamoun M B, Benaini R. Short: "A case study of the perfor-mance of an OpenFlow controller". In: Proceedings of 
the 2nd International Conference on Lecture Notes in Computer Science (LNCS), 2014: 330-334.

[14] Bing Xiong, Kun Yang, Jinyuan Zhao, Wei Li, Keqin Li, "Performance evaluation of OpenFlow-based software-defined networks based on Queuing model" , Elsevier Volume 102, 19 June 2016, Pages 172-185

[15] Michael Jarschel, Simon Oechsner, Daniel Schlosser, Rastin Pries, Sebastian Goll, Phuoc Tran-Gia, "Modeling and Performance Evaluation of an OpenFlow Architecture", 2011 ITC.

[16] D. Gross, J. F. Shortle, J. M. Thompson, and C. M. Harris, "Fundamentals of Queuing theory". John Wiley \& Sons, 2013.

[17] J. L. Boudec and P. Thiran, "Network Calculus: A Theory of Deterministic Queuing Systems for the Internet". Berlin, Heidelberg: Springer- Verlag, 2001.

[18] Y. Luo, P. Cascon, E. Murray, and J. Ortega. "Accelerating OpenFlow switching with network processors". In Proceedings of the $5^{\text {th }}$ ACM/IEEE Symposium on Architectures for Networking and Communications Systems, ANCS '09, pages 70-71, New York, NY, USA, 2009. ACM.

[19] A. Tootoonchian, S. Gorbunov, Y. Ganjali, M. Casado, and R. Sherwood. "On controller performance in software defined networks". In USENIX Workshop on Hot Topics in Management of Internet, Cloud, and Enterprise Networks and Services (Hot ICE), 2012.

[20] S. Yeganeh, A. Tootoonchian, and Y. Ganjali. "On scalability of software defined networking. Communications Magazine", IEEE, 51(2):136- 141, February.

[21] Bing Xiong, Kun Yang, Jinyauan Zhao, Wei Li, Keqin L I, "Performance evaluation of OpenFlow based software defined networks based on Queuing model", JAN 2016.

[22] L. Kleinrock, Queuing Systems - Volume 1: Theory. Wiley- Inter science, 1975.

[23] Edward D. Lazowska, John Zahorjan, G. Scott Graham, and Kenneth C. Sevcik, "An Overview of Queuing Network Modelling", 1984.

[24] Jain R, Routhier S A. Packet trains: measurements and a new model for computer network traffic. IEEE Journal on Selected Areas in Communications, 1986, 4(6): 986-995.

[25] Choi B D, Choi D I, Lee Y, Sung D K, "Priority Queuing system with fixed-length packet-train arrivals", IEE Proceedings Communications, 1998, 145(5): 331-336.

[26] Paxson V, Floyd S, "Wide area traffic: the failure of Poisson modeling" IEEE/ACM Transactions on Networking (ToN), 1995, 3(3): 226-244.

[27] Bing Xiong, "Performance Evaluation of OpenFlow-based Software-defined Networks Based on Queuing Model", IEEE, January 17, 2016.

[28] Jonathan Marchini, “The Poisson Distribution" November 10, 2008.

[29] Guodong Wang, Jun Li, Xiangqing Chang, "Modeling and Performance Analysis of the Multiple Controllers' Approach in Software Defined Networking", IEEE 23rd International Symposium on Quality of Service (IWQoS), 2015.

[30] M. Jarschel, S. Oechsner, D. Schlosser, R. Pries, S. Goll, and P. Tran-Gia, "Modeling and performance evaluation of an OpenFlow architecture," in Proceedings of the 23rd International Tele traffic Congress. ITCP, 2011, pp. 1-7.

[31] L. Kleinrock and F. A. Tobagi, "Packet switching in radio channels: Part I-carrier sense multiple-access modes and their throughput-delay characteristics", Communications, IEEE Transactions on, vol. 23, no. 12, pp. 1400-1416, 1975.

[32] R. L. Cruz, "A calculus for network delay. i. network elements in isolation," Information Theory, IEEE Transactions on, vol. 37, no. 1, pp. 114-131, 1991.

[33] Y. Jiang and Y. Liu, Stochastic network calculus. Springer, 2008, vol. 1.
[34] Siamak Azodolmolky, "Software defined netwoking a comprenshive survey", IEEE,2015

[35] Thomas Ball and Nikolaj Bjørner, Aaron Gember, Shachar Itzhaky,VeriCon: Towards Verifying Controller Programs in Software-Defined Networks, PLDI '14, June 9 - 11 2014,

[36] Canini, M., Venzano, D., Peres, P., Kostic, D., And Rexford, J. A Nice Way To Test Openflow Applications. In Nsdi (2012).

[37] Sethi, D., Narayana, S., And Malik, S. Abstractions for model checking sdn controllers. In FMCAD (2013).

[38] NELson, T., Ferguson, A. D., Scheer, M. J. G., And Krishnamurthi, S. "A balance of power: Expressive, analyzable controller programming. In 11th USENIX Symposium on Networked Systems Design and Implementation (NSDI '14) (2014).

[39] Tkachova E., Demchenko L., Saied Halawa Fawaz. Verification of telecommunication protocols based on formal methods [Electronic Resource] International journal of intelligent information system 2013; 2(1) pages 1-10, February 20, $2013-$ Mode of access:

[40] Amer Tahseen Salameh Abu-Jassar, "Mathematical Tools for SDN Formalization and Verification", Second International Scientific-Practical Conference, PIC S\&T'2015

[41] J. Choi, I. Lee, and H. Xie, "The specification and schedulability analysis of real-time systems using ACSR," in Proc. IEEE RealTime Systems Symp., Dec. 1995.

[42] Sooel Son, Seungwon Shin, Vinod Yegneswaran, Phillip Porras, Guofei Gu, "Model Checking Invariant Security Properties in OpenFlow" Communication and Information Systems Security Symposium, IEEE ICC 2013

[43] https://en.wikipedia.org/wiki/Satisfiability_modulo_theories

[44] https://sourceforge.net/projects/cbench/

[45] Yutaka Yakuwa, "EFficient Model Checking Of Openlow Networks Using SDPOR-Ds"

[46] http://archive.openflow.org/wk/index.php/OFRewind

[48] https://www.sdxcentral.com/projects/oflops/

[49] http://www.uppaal.org/ 